\title{
REPORTE DE UNA POBLACIÓN SILVESTRE DE SEDUM MORGANIANUM E. WALTHER (CRASSULACEAE)
}

H. David Jimeno-Sevilla ${ }^{1}$, Miguel Cházaro-Basáñez ${ }^{2}$ y Amparo Albalat-Botana ${ }^{3}$

${ }^{1}$ Universidad Veracruzana, Centro de Investigaciones Tropicales, interior de la Ex hacienda Lucas Martín, calle Araucarias s/n, colonia Periodistas,

91019 Xalapa, Veracruz, México.bpdjimeno@yahoo.com.mx

${ }^{2}$ Universidad de Guadalajara, Centro Universitario de Ciencias Sociales y Humanidades, Departamento de Geografía y Ordenación Territorial, Apdo. postal

30,45101 Zapopan, Jalisco, México. chazaro55@hotmail.com

${ }^{3}$ Colegio de Posgraduados, Campus Veracruz, km. 88.5 de la carretera federal

Xalapa-Veracruz. 421, predio Tepetates, Manlio Fabio Altamirano, Veracruz,

México.amparoalbalat@hotmail.com

\section{RESUMEN}

Se reporta el hallazgo de Sedum morganianum E. Walther (cola de borrego) en su estado silvestre. Esta especie fue descrita y nombrada a partir de material cultivado y a más de 70 años de su publicación, no se conocían plantas de origen silvestre. Se encontró en dos barrancas del municipio de Tenampa, en el centro de Veracruz, en el oriente de México, donde crece sobre paredes verticales de arenisca con bosque tropical caducifolio. Por su distribución conocida se piensa que se trata de un endemismo estrecho.

Palabras clave: Crassulaceae, endemismo, México, Sedum, Veracruz.

\section{ABSTRACT}

Over 70 years after its original description, Sedum morganianum E. Wather, the "donkey tail", has been at last found wild in two ravines at Tenampa county, in central Veracruz, in eastern Mexico. It grows on vertical cliffs of igneous rock in the Tropical Deciduous Forest zone. For its restricted geographic distribution it should be regarded as a micro endemic species.

Key words: Crassulaceae, endemism, Mexico, Sedum, Veracruz. 


\section{INTRODUCCIÓN}

Sedum morganianum E. Walther es una planta de ornato ampliamente cultivada en México y en otros países, por su vistoso hábito colgante, la coloración azul glauca de sus hojas y tallos, sus flores rojizas y su fácil propagación vegetativa.

Sin embargo, pese a su vasta distribución como planta de ornato, por siete décadas se desconoció la existencia de ejemplares de origen silvestre. Uhl (1980) señaló: "No definite locality is yet known for it in the wild, but, like S. morganianum, it is suspected that it may be native somewhere on the eastern slopes of Mount Orizaba or nearby."

Varios autores suponen que la distribución natural de Sedum morganianum se encuentra en el oriente de México (t’Hart y Bleij, 2003; Meyrán y López, 2003), basados en que el material tipo provenía de una planta que Eric Walther obtuvo de un vivero de la ciudad de Coatepec (Walther, 1938) y a que Clausen (1959) observó que era frecuente encontrarlo cultivado en Coscomatepec y Orizaba, ciudades de la región central del estado de Veracruz.

Al igual que Sedum morganianum, existen otros miembros de Crassulaceae descritos a partir de plantas de cultivo, de las que se desconocía su hábitat silvestre, y que posteriormente se encontraron en su ambiente natural; tal es el caso de Graptopetalum superbum (Kimnach) Acevedo-Rosas, G. pentandrum Moran y Cremnophila linguifolia (Lem.) Moran. A pesar de que el conocimiento de los representantes de esta familia se ha incrementado en México en los últimos años, todavía hay especies de las cuales se desconoce su hábitat en estado silvestre, como Graptopetalum paraguayense ssp. paraguayense, (Brown) E. Walther, Sedum burrito Moran, Sedum mexicanum Britton y Echeveria prolifica Moran \& J. Meyrán.

Aunque el estado de Veracruz es una de las regiones de nuestro país más estudiadas en el aspecto botánico, las especies de la familia Crassulaceae de la entidad están pobremente representadas en los herbarios mexicanos. En su territorio existen zonas poco conocidas con gran potencial de exploración para el grupo, como la Sierra de Huayacocotla en el norte de Veracruz, la zona árida del Cofre de Perote y el Pico de Orizaba, los alrededores de la ciudad de Córdoba, la sierra de Zongolica y las barrancas profundas del centro del estado (Jimeno-Sevilla, 2008), de las cuales recientemente se han dado a conocer diversas novedades.

En febrero de 2008, durante un estudio prospectivo, se visitó el rancho Bellreguard de Sochiapa en el Municipio de Tenampa, en el centro de Veracruz, donde el señor Carlos Ros nos mostró una planta de la familia Crassulaceae que crece en la zona. La especie fue determinada como Sedum morganianum E. Walther. Conside- 
rando que no se tenían reportes de poblaciones silvestres, se optó por dar a conocer tal hallazgo.

\section{DESCRIPCIÓN DEL MATERIAL COLECTADO}

Sedum morganianum E. Walther. Cact. Succ. J. 10: 35-37. 1938. Tipo obtenido de plantas cultivadas en San Francisco de material originario de México, Veracruz, municipio de Coatepec, E. Walther 35/23. CAS! (Figs. 1 y 2).

Planta subarbustiva; tallos péndulos de $90 \mathrm{~cm}$ o más de largo, de $3.5-4 \mathrm{~mm}$ de diámetro, de color rojizo a verde, glaucos; raíces fibrosas; hojas dispuestas en espiral a lo largo del tallo, imbricadas y adpresas cuando jóvenes, separándose del tallo en la madurez, lanceoladas o falcadas en contorno, semirrollizas, con el margen entero, de 1.8-2.1 cm de largo, de 6-8.5 $\mathrm{mm}$ de ancho y de 4-5 $\mathrm{mm}$ de grosor, la cara superior cóncava-aquillada a convexa, la cara inferior convexa, de color verde azuloso, glaucas, ápice agudo, usualmente el ápice y los bordes rojizos, caedizas; inflorescencia en forma de corimbo, 1-2 por rama, tallos florales terminales o laterales, de $7 \mathrm{~mm}$ de largo y de $3 \mathrm{~mm}$ de diámetro, de color verde azuloso, pruinoso, con 6-7 flores, brácteas alternas, lanceoladas a oblongo-lanceoladas, persistentes, convexas en la cara inferior y aplanadas en la superior, hasta de $12 \mathrm{~mm}$ de largo, $3 \mathrm{~mm}$ de ancho y $2 \mathrm{~mm}$ de grosor, de color verde azuloso, pruinosas, ápice agudo; bracteolas oblongo-lanceoladas, de 1-2.5 $\mathrm{cm}$ de largo, de 0.8-1.8 $\mathrm{mm}$ de ancho, ápice acuminado, de color verde pálido, pedicelo engrosándose hacia la base del cáliz, de 1.75-2 cm de largo y de $1.6 \mathrm{~mm}$ de diámetro, de color verde pálido, ascendente; cáliz de 5 sépalos unidos en la base formando un tubo de $4 \mathrm{~mm}$ diámetro y $2.2 \mathrm{~mm}$ de largo, lóbulos lanceolados, acuminados, subiguales, ascendentes, adpresos, de 4-5 mm de largo y de 2.4-3 $\mathrm{mm}$ de ancho, con ambas caras aplanadas, de color verde; corola subcampanulada de hasta $8 \mathrm{~mm}$ de largo y de $6.7 \mathrm{~mm}$ de diámetro, de color rojo escarlata, con el interior rojizo-rosado; pétalos 5, ovados, de $8 \mathrm{~mm}$ de largo, $4 \mathrm{~mm}$ de ancho, libres, erectos ápice acuminado; estambres 10 , 5 epipétalos de $5 \mathrm{~mm}$ de largo adnados al pétalo cerca de $2 \mathrm{~mm}$ y 5 antesépalos libres de $5.5 \mathrm{~mm}$ de largo, de color rojizo; anteras de color amarillo; nectarios de $1.7 \mathrm{~mm}$ de ancho, de color rojizo; carpelos 5, libres entre sí, de $4 \mathrm{~mm}$ de largo y de $1.8 \mathrm{~mm}$ de ancho cada uno, erectos, estilos de color rojizo-rosado, de $2 \mathrm{~mm}$ de largo; folículos de $3.8 \mathrm{~mm}$ de largo, ascendentes, de color café rojizo, semillas de color café, numerosas. 

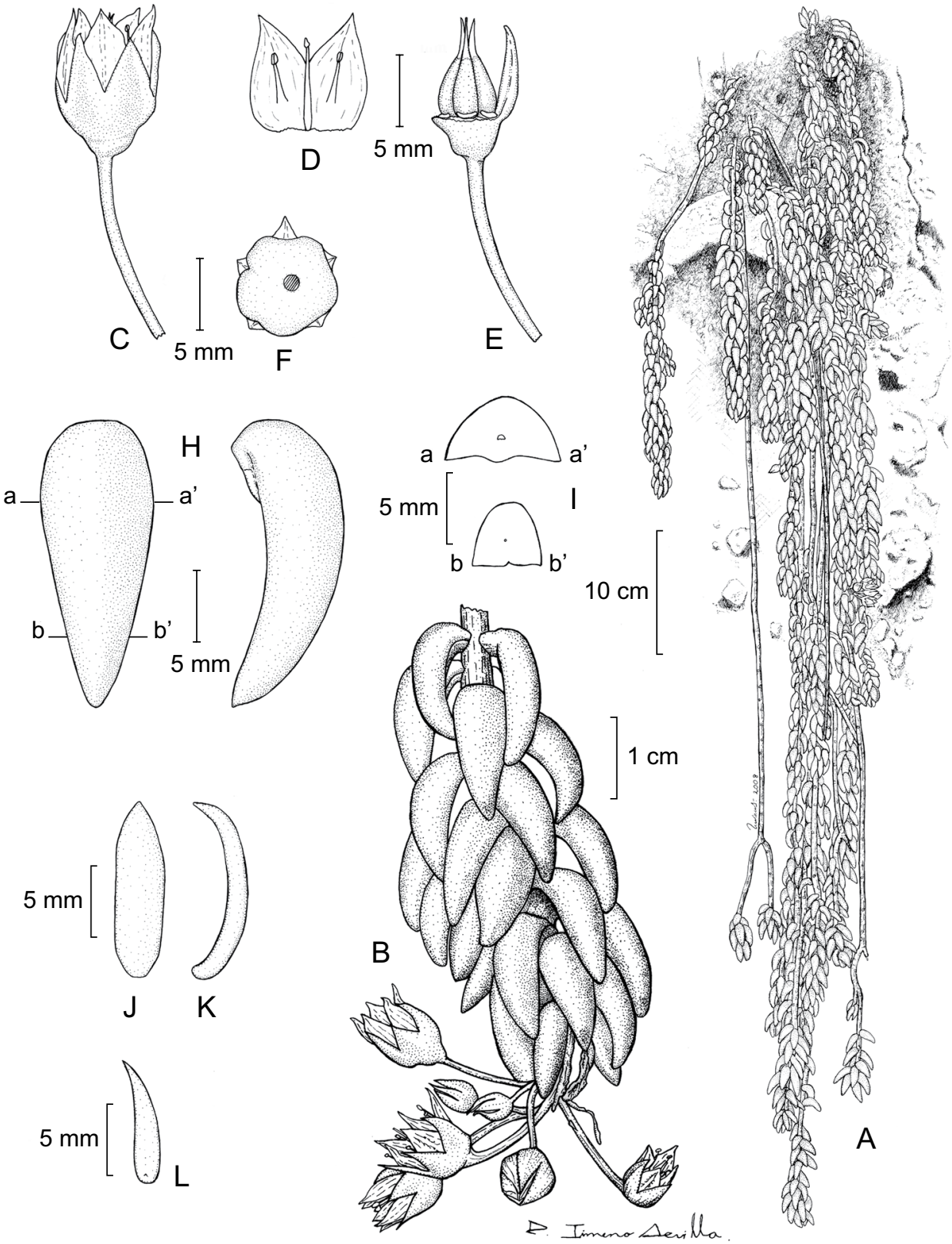

Fig. 1. Sedum morganianum. A. hábito; B. detalle de inflorescencia; C. flor; D. interior de los pétalos; E. gineceo; F. vista inferior de la flor; G. y H. vista dorsal y lateral de la hoja; I. cortes trasversales de la hoja; J. y K. vista dorsal y lateral de la bráctea; L. vista dorsal de la bracteola. (con base en D. Jimeno-Sevilla, A. Albalat \& C. Ros 425). 

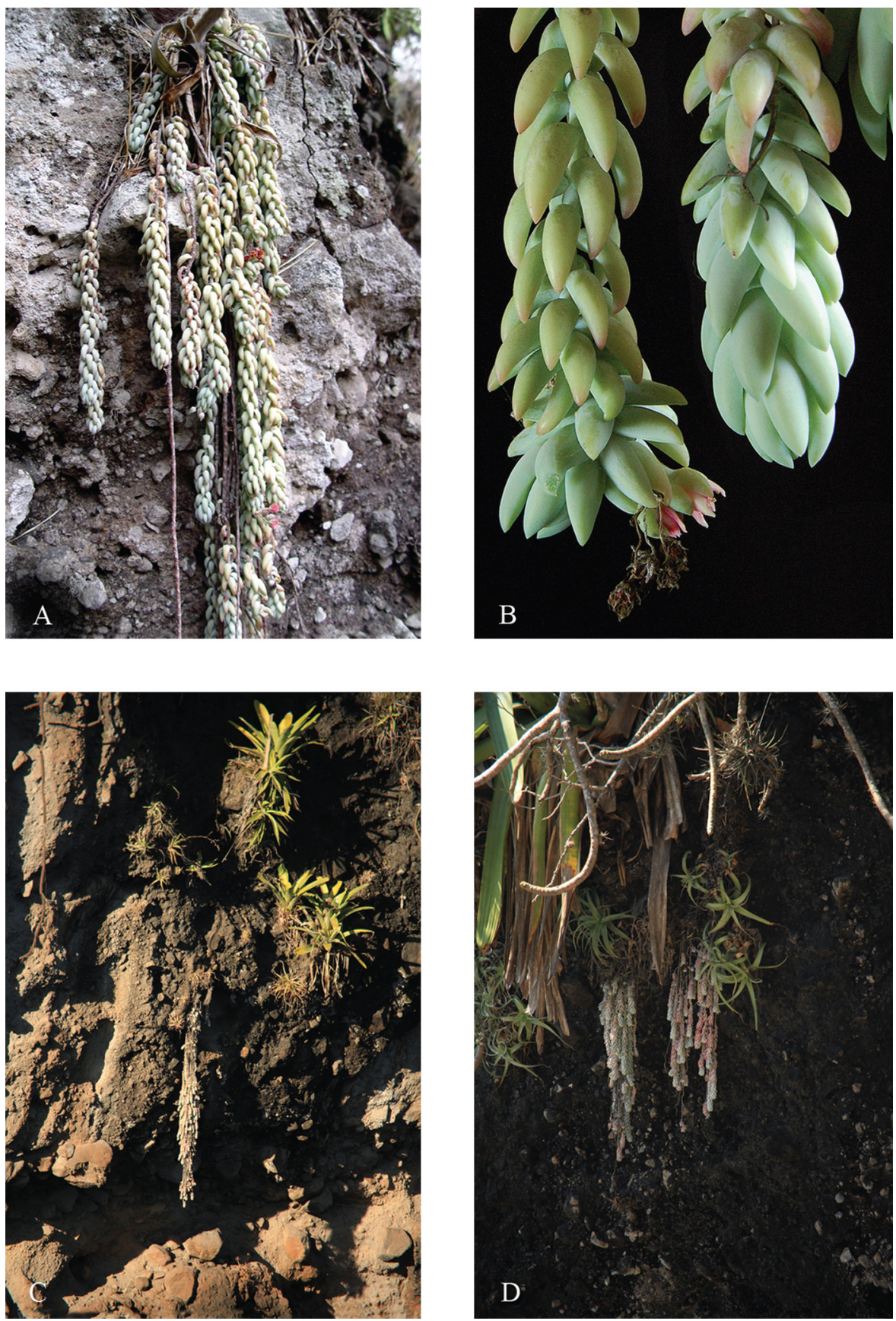

Fig. 2. Sedum morganianum. A. hábito; B. detalle de la inflorescencia; C. y D. hábito. 
Distribución y hábitat: Sedum morganianum se conoce de la región centro del estado de Veracruz, de dos cañadas profundas que corren en dirección O-E, la barranca de Mayatla y la de Ixcacotitla, pertenecientes al municipio de Tenampa. Crece sobre paredes verticales de arenisca conglomerada del Cuaternario (Anónimo, 2002a) de muy difícil acceso, en exposición N y S, en un reducido intervalo altitudinal que va de los 600 a los $700 \mathrm{~m}$, por lo que se considera un endemismo estrecho. La población es numerosa, se observan cientos de individuos en los cantiles.

La vegetación en la porción superior y paredes de las cañadas corresponde al bosque tropical caducifolio y al fondo de estas se encuentra el bosque tropical subperennifolio. En el área existe una gran cantidad de plantas suculentas como Mammillaria sartorii J. A. Purpus, Selenicereus spp., Echeveria carnicolor (Baker) E. Morren, Sedum nussbaumerianum Bitter, Sedum aff. hemsleyanum Rose, Beaucarnea recurvata Lem. Opuntia decumbens Salm-Dyck, Hechtia myriantha Mez, H. purpusii Brandegee, Tillandsia grandis Schltdl. y Agave pendula Schnittsp., entre otras.

Fenología: florece y fructifica de febrero a julio.

Nombre común: en la región centro de Veracruz se conoce como "cola de borrego" y "cola de burro".

Material observado: México, Veracruz, municipio de Tenampa, Barranca de Mayatla, Rancho Bellreguard de Sochiapa, Febrero 2008 (con flores). Rupícola, 700 m s.n.m. D. Jimeno-Sevilla, A. Albalat \& C. Ros 425 (IEB, MEXU, XAL).

\section{DISCUSIÓN}

El material colectado en campo se revisó y comparó con el ejemplar tipo, con la descripción original y con plantas cultivadas que posiblemente proceden del mismo material de donde se obtuvo el ejemplar tipo. Las muestras de origen silvestre presentan medidas menores en el grosor del tallo, el tamaño de las hojas y el largo del pedicelo. Parte de la planta colectada en el campo se mantiene en cultivo en Coatepec, Veracruz, en la cual se observa un incremento de tamaño en las hojas y el tallo, con lo cual se asemeja a las medidas de la descripción de Walther (1938). Un carácter nunca antes mencionado para la especie es la unión de los filamentos del epipétalo, los cuales están adnados en el pétalo, (aproximadamente $2 \mathrm{~mm}$ ), mientras que los antesépalos se mantienen libres. Dentro de los taxa de Crassulaceae mexicanos que 
comparten esta característica se encuentran S. burrito Moran (Moran, 1977), y $S$. corynephyllum Fröd., así como los géneros Echeveria DC, Dudleya Britton \& Rose y Pachyphytum Link, Klotzsch \& Otto.

La especie más cercana a Sedum morganianum aparentemente es $S$. burrito Moran, por su hábito colgante, el color de las hojas, el tipo de inflorescencia y la forma y color de las flores; se diferencian en el largo de los pedicelos, el tamaño de las flores y en la forma de sus hojas. S. burrito se describió en 1977, con base en plantas cultivadas procedentes de Coatepec, Veracruz (Moran, 1977). Dada la semejanza morfológica entre estas dos especies, es probable que $S$. burrito se distribuya en ambientes similares, por lo que los esfuerzos para encontrarla silvestre deben estar dirigidos a dichas áreas.

Con la finalidad de conocer mejor la distribución de $S$. morganianum y establecer acciones de conservación, resultaría interesante buscar más poblaciones en la región central de Veracruz, en condiciones fisiográficas e intervalos altitudinales similares. Se propone que Sedum morganianum sea incluida en la NOM-059-ECOL2001 (Anónimo, 2002b) bajo la categoría de protección especial y endémica, debido a lo restringido de su distribución.

\section{AGRADECIMIENTOS}

Agradecemos al Señor Carlos Ros por el apoyo para la colecta del material, así como por su interés, entusiasmo y disposición para realizar el trabajo de campo; al Centro de Investigaciones Tropicales (CITRO) de la Universidad Veracruzana, y la Comisión del estado de Veracruz para la Conmemoración de la Independencia Nacional y de la Revolución Mexicana por el apoyo económico brindado para realizar las salidas de campo y la toma de fotografías, dentro del proyecto "Atlas de la Flora Endémica, Notable y en Peligro de Extinción de Veracruz"; a Pablo Carrillo, Evelia Botana Montenegro y Mónica Vargas por la revisión del manuscrito y sus valiosos comentarios. Finalmente a Gerardo Sánchez Vigil, por las fotografías (figura $2 \mathrm{C}$ y D).

\section{LITERATURA CITADA}

Anónimo, 2002a. Carta geológica E14-3, 1:250000. Veracruz. Instituto Nacional de Estadística, Geografía e Informática. Aguascalientes, Ags. 
Anónimo, 2002b. Norma Oficial Mexicana NOM-059-ECOL-2001. Protección ambiental -especies nativas de México de flora y fauna silvestres- categorías de riesgo y especificaciones para su inclusión, exclusión o cambio. Lista de especies en riesgo. Secretaría de Medio Ambiente y Recursos Naturales. Diario Oficial de la Federación. México, D.F., miércoles 6 de marzo.

Clausen, R. T. 1959. Sedum of the Trans-Mexican Volcanic Belt: an exposition of taxonomic methods. Cornell University Press. Ithaca, New York. 380 pp.

Jimeno-Sevilla, H. D. 2008. El género Echeveria (Crassulaceae) en Veracruz, México. Tesis de licenciatura. Facultad de Biología, Universidad Veracruzana, Xalapa, Veracruz. $136 \mathrm{pp}$.

Meyrán, J. y L. López. 2003. Las crasuláceas de México. Sociedad Mexicana de Cactología, A.C. México, D.F. 234 pp.

Moran, R. 1977. Sedum burrito, a new species from Eastern Mexico. Cact. Succ. J. 49: 266268.

t'Hart, H. y B. Bleij. 2003. Sedum. In: Eggli, U. (ed.). Illustrated handbook of succulent plants: Crassulaceae. Springer. Berlin. pp. 235-332.

Uhl, C. H. 1980. Chromosomes of Mexican Sedum III. Sections Centripetala, Fruticisedum and other woody species. Rhodora (US) 82: 377-402

Walther, E. 1938. Notes on Crassulaceae. Cact. Succ J. 10: 35-37. 